\title{
EXCITON IN 2D CUBIC INCLUSION IN HEXAGONAL GaN
}

\author{
M. SUFFCZYŃSKI \\ Institute of Physics, Polish Academy of Sciences \\ Al. Lotników 32/46, 02-668 Warsaw, Poland \\ R. STĘPNIEWSKI AND J.M. BaRANOWSKI
}

Institute of Experimental Physics, Warsaw University, Hoża 69, 00-681 Warsaw, Poland

\begin{abstract}
An inclusion of cubic $\mathrm{GaN}$ in an otherwise hexagonal matrix is considered to be equivalent to an effective quantum well. The exciton binding energy in a quantum well of a finite potential barrier height in the conduction and valence bands is calculated in the effective mass approximation with a variational envelope function type of Bastard and Takagahara. The exciton binding energy and the energy of exciton recombination line are computed, as a function of the well width, for realistic potential barrier heights and band-offset ratios.
\end{abstract}

PACS numbers: 73.20.Dx, 78.66.-w, 61.72.Nn

\section{Introduction}

Gallium nitride crystallizes in the wurtzite structure. However, an epitaxial metastable zinc-blende type cubic structure can also be grown [1]. The optical energy gap of the cubic phase $E_{\text {gap }}=3.302 \mathrm{eV}$ [2] was found to be about $0.2 \mathrm{eV}$ smaller than the gap of the hexagonal phase $[3,4]$.

In the case of gallium nitride grown epitaxially on sapphire, due to a large lattice mismatch between the substrate and the GaN layer, numerous extended defects are present close to the interface [5]. An interesting situation arises in the case of formation of stacking faults in the wurtzite structure e.g. due to the insertion of an extra atomic plane. This extra plane can be treated as a flat inclusion of the cubic GaN in the hexagonal GaN matrix. Such inclusions, due to one or several additional planes have been observed in $\mathrm{GaN}$ by high resolution transmission microscopy $[5,6]$, and can be considered as producing an effective $2 \mathrm{D}$ quantum well.

In this paper the ground state energy of an exciton confined in the well is computed. The exciton binding energy in such a quantum well of finite barrier height in the conduction and in the valence band is calculated in the effective mass approximation, with a variational envelope function type of Bastard [7-9] and Takagahara $[10,11]$. Continuity of the envelope function and of the current density at the well boundary is assumed [9-12]. 


\section{Variational envelope function of exciton ground state in quantum well}

The exciton binding energy in a quantum well of width $L$, of a finite barrier height $E_{\mathrm{c}}$ in the conduction band, and $E_{\mathrm{v}}$ in the valence band, is calculated using the variational envelope function [7-12]

$$
\Phi\left(\rho, z_{\mathrm{e}}-z_{\mathrm{h}}, \lambda\right)=A^{-1} f_{\mathrm{e}}\left(z_{\mathrm{e}}\right) f_{\mathrm{h}}\left(z_{\mathrm{h}}\right) g\left(\rho, z_{\mathrm{e}}-z_{\mathrm{h}}, \lambda\right)
$$

with

$$
g(\rho, z, \lambda)=\exp (-r / \lambda)
$$

where $\rho=\left(x^{2}+y^{2}\right)^{1 / 2}, r=\left(\rho^{2}+z^{2}\right)^{1 / 2}$,

$$
\begin{gathered}
f_{\mathrm{e}}(z)=\cos (k z), V_{\mathrm{e}}(z)=0, f_{\mathrm{h}}(z)=\cos \left(k_{\mathrm{h}} z\right), V_{\mathrm{h}}(z)=0, \\
\quad \text { for }|z|<L / 2, \\
f_{\mathrm{e}}(z)=B_{\mathrm{e}} \exp (-q z), V_{\mathrm{e}}(z)=E_{\mathrm{c}}, f_{\mathrm{h}}(z)=B_{\mathrm{h}} \exp \left(-q_{\mathrm{h}} z\right), V_{\mathrm{h}}(z)=E_{\mathrm{v}}, \\
\text { for }|z|>L / 2 .
\end{gathered}
$$

The energy gap $E_{\text {gap }}$ is not shown here, since it does not affect the exciton binding energy.

3. Variational calculation of exciton ground state in quantum well is $[7-12]$

The effective mass Hamiltonian of an exciton in a rectangular quantum well

$$
H=-\hbar^{2} \Delta_{\mathrm{e}} / 2 m_{\mathrm{e}}-\hbar^{2} \Delta_{\mathrm{h}} / 2 m_{\mathrm{h}}-e^{2} / \varepsilon r+V_{\mathrm{e}}\left(z_{\mathrm{e}}\right)+V_{\mathrm{h}}\left(z_{\mathrm{h}}\right)
$$

with the Laplacians $\Delta_{\mathrm{e}}$ and $\Delta_{\mathrm{h}}$, for the electron and hole, respectively.

The conduction and the valence band edge effective masses are $m_{\mathrm{e}}$ and $m_{\mathrm{h}}$ inside the well, and the conduction and the valence band edge effective masses are $m_{\mathrm{c}}$ and $m_{\mathrm{v}}$ outside the well, respectively. The exciton reduced mass $\mu=$ $1 /\left(1 / m_{\mathrm{e}}+1 / m_{\mathrm{h}}\right)$, and the dielectric constant $\varepsilon$ inside the quantum, well, determine the exciton effective Rydberg $R y d=\mu e^{4} / 2 \varepsilon^{2} \hbar^{2}$ and the exciton effective Bohr radius $a_{\mathrm{B}}=\varepsilon \hbar^{2} / \mu e^{2}$, which are used as units of energy and length, respectively.

Analytical expressions have been derived for the envelope function norm $A$ and the expectation value of the Hamiltonian of the exciton in its ground state. The optimized value of the variational parameter $\lambda$ was found by numerical interpolation. For quantum well barrier heights $E_{\mathrm{c}}$ and $E_{\mathrm{v}}$ much higher than the effective Rydberg energy the binding energy $E_{\mathrm{B}}$ of the ground state of exciton in the quantum well is computed [7-12] as

$$
E_{\mathrm{B}}=A^{-2} U-\lambda^{-2} / 2 \mu
$$

with analytical expressions given [10] in terms of the following parameters:

$$
b=\pi \lambda / L \text { and } e_{2}=\exp (-2 L / \lambda)
$$

by

$$
\begin{aligned}
A^{2}= & L\left[1+\left(1+b^{2}\right)^{-2}\left(1+b^{4} e_{2}\right) / 2\right] \\
& +\lambda b^{4}\left(7+3 b^{2}\right)\left(1+b^{2}\right)^{-3}\left(e_{2}-1\right) / 4
\end{aligned}
$$




$$
U=(L / \lambda)\left\{1+\left[1+b^{2}\left(1+b^{2}\right)^{-1}\left(e_{2}-1\right)\right]\left(1+b^{2}\right)^{-1} / 2\right\} .
$$

For general values of quantum well barrier height expressions for $A$ and $U$ are too long to be reproduced here, and can be supplied on request.

The band-offset ratios for the wurtzite-cubic GaN interface have not been estimated. However, for slightly different crystal structures of the same chemical compound one can expect small differences of the electron energy for the occupied states in the valence band. Therefore, we assume the band-offset ratio $E_{\mathrm{c}}: E_{\mathrm{v}}=$ $80: 20$, which leads to the conduction and valence band barrier heights $E_{\mathrm{c}}=$ $0.16 \mathrm{eV}$ and $E_{\mathrm{v}}=0.04 \mathrm{eV}$, respectively. The exciton binding energy is computed for typical quantum well widths, using the following parameters [4]: $m_{\mathrm{e}}=m_{\mathrm{c}}=$ $0.22 m_{0}, m_{\mathrm{h}}=m_{\mathrm{v}}=0.75 m_{0}, \varepsilon=9.6$. These parameters give $R y d=25.1 \mathrm{meV}$ and $a_{\mathrm{B}}=29.9 \AA$. The computations are performed for the quantum well width varying from $5 \AA$ in the range up to $100 \AA$.

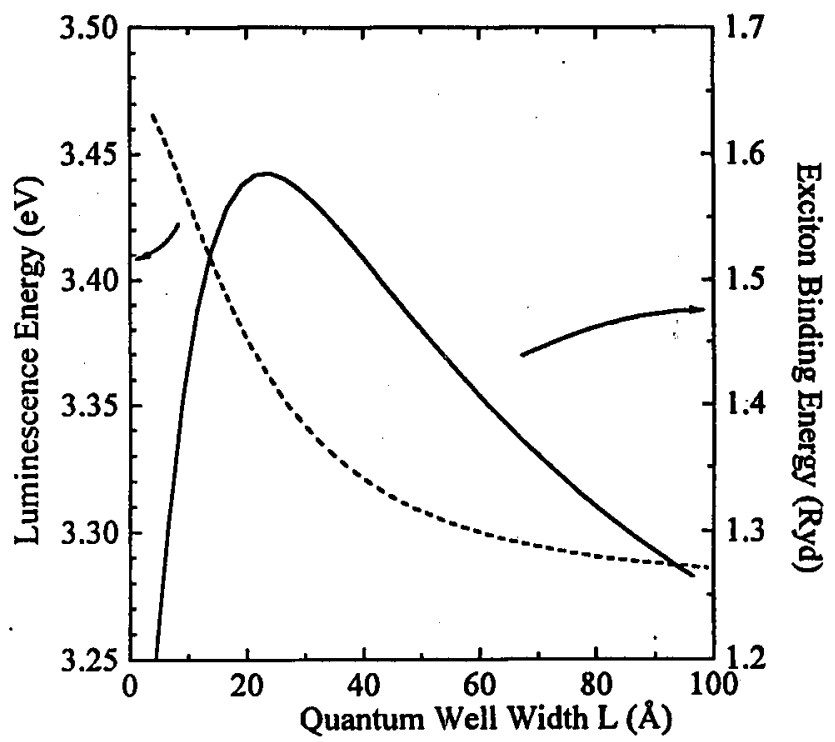

Fig. 1. Solid line - the computed binding energy of the exciton confined in the $\mathrm{GaN}$ (cub)/GaN(wurtz) quantum well, as a function of the well width ( $R y d=25.1 \mathrm{meV}$ ). Dashed line - the computed exciton recombination energy.

Figure 1 shows the computed binding energy of the exciton confined in the $\mathrm{GaN}(\mathrm{cub}) / \mathrm{GaN}$ (wurtz) quantum well with the hole from the highest subband. The exciton binding energy attains its largest value for the quantum well width of about $25 \AA$. It decreases in quantum well of small width when more wave function leaks out of the well. The computed exciton recombination energy, resulting from its confinement and the energy position of the lowest sublevel of the electron and the highest sublevel of the hole in the quantum well, is indicated, as a function of the well width, by the dashed line. It is seen that in the energy ranme betwiese 
$3.30 \mathrm{eV}$ and $3.45 \mathrm{eV}$ a luminescence line due to the cubic inclusions in wurtzite GaN may be expected.

\section{References}

[1] R.C. Powell, N.E. Lee, Y.W. Kim, J.E. Greene, J. Appl. Phys. 73, 189 (1993).

[2] G. Ramirez-Flores, H. Navarro-Contreras, A. Lastras-Martinez, R.C. Powell, J.E. Greene, Phys. Rev. B 50, 8433 (1994).

[3] K.P. Korona, A. Wysmolek, K. Pakuła, R. Stępniewski, J.M. Baranowski, I. Grzegory, B. Eucznik, M. Wróblewski, S. Porowski, Appl. Phys. Lett. 69, 788 (1996); S. Nakamura, M. Senoh, S. Nagahama, N. Iwasa, T. Yamada, T. Matsushita, Y. Sugimoto, H. Kiyoku, Appl. Phys. Lett. 70, 616 (1977).

[4] R. Stępniewski, A. Wysmołek, Acta. Phys. Pol. 90, 681 (1996).

[5] P. Vennegues, B. Beaumont, JEOL News 32, 20 (1996).

[6] Z. Lilienthal-Weber, C. Kisielowski, S. Ruvimov, Y. Chen, J. Washborn, I. Grzegory, M. Boćkowski, J. Jun, S. Porowski, J. Electron. Malter. 25, 1545 (1996).

[7] G. Bastard, Phys. Rev. B 24, 4714 (1981).

[8] G. Bastard, E.E. Mendez, L.L. Chang, L. Esaki, Phys. Rev. B 26, 1974 (1982).

[9] W. Liu, J.J. Quinn, Phys. Rev. B 31, 2348 (1985).

[10] T. Takagahara, Phys. Rev. $B$ 31, 6552 (1985).

[11] M. Kumagai, T. Takagahara, Phys. Rev. B 40, 12359 (1989).

[12] R.L. Greene, K.K. Bajaj, Phys. Rev. B 31, 6498 (1985). 